\title{
Central Airway Obstruction due to Mediastinal Malignant Lymphoma Requiring Venovenous-Extracorporeal Membrane Oxygenation
}

\author{
Yoshihiro Ueda Ichiro Hirayama Ryohei Horie Kent Doi \\ Naoto Morimura \\ Department of Acute Medicine, The University of Tokyo Hospital, Tokyo, Japan
}

\section{Keywords}

Airway obstruction - Mediastinal tumor - Extracorporeal membrane oxygenation

\begin{abstract}
Extracorporeal membrane oxygenation (ECMO) therapy might be controversial when patients with advanced malignant disease develop heart or lung failure refractory to conventional management. Especially as for the hematological malignancy patients, the induction of ECMO therapy must be considered carefully, since it is often associated with bleeding complications or infectious diseases. Here, we report a case of life-threatening airway obstruction requiring ECMO. The trachea of the patient was narrowed by an anterior mediastinal tumor too highly to ventilate both lungs, so she had to be connected to venovenous-ECMO (VV-ECMO) before pathological examination and radical treatment were planned. During the intensive care with ECMO, she was diagnosed with malignant lymphoma and the chemotherapy was started. The chemotherapy brought such an immediate result that the trachea regained its patency and ECMO was disconnected 9 days after the initiation of the chemotherapy. Then, the patient was able to leave the intensive care unit with no sequelae. Central airway obstruction is a lifethreatening situation, in which prompt decisions are essential. On the other hand, if the airway is impaired by hematological malignancy, it might be generally challenging to consider ECMO
\end{abstract}


as a bridge until the chemotherapy takes an effect. The prognosis of hematological malignancy differs according to its subtype or stage, so it may be reasonable to take ECMO therapy into consideration for some population.

(C) 2019 The Author(s)

Published by S. Karger AG, Basel

\section{Introduction}

It is challenging to induce extracorporeal membrane oxygenation (ECMO) in case of severe respiratory failure related to hematological malignancy because such patients are vulnerable to several life-threatening complications [1-3]. Here, we report the case of central airway obstruction caused by anterior mediastinal lymphoma successfully managed with venovenous-ECMO (VV-ECMO).

\section{Case Report}

A 45-year-old woman had had complaint of dyspnea for several days and visited another hospital. She presented remarkable stridor on auscultation, and she could not lay on her back due to difficulty in breathing. She was referred to our hospital and was intubated soon after arrival because she was considered to be in danger of airway obstruction. Chest X-ray and CT scans revealed that the trachea was compressed by an anterior mediastinal tumor (Fig. 1a, b), and the subsequent bronchoscopy showed that the trachea was markedly narrowed just above the carina (Fig. 2). It was possible to ventilate at that moment, though the airway was still narrowed distal to the endotracheal tube. She was admitted to the intensive care unit and standard ventilator support was started. Then, the respiratory status and hemodynamics were stabilized temporarily. However, on the second day, the tidal volume decreased gradually and it became almost impossible to ventilate. Arterial blood gas analysis revealed a $\mathrm{pH}$ of 7.22, $\mathrm{PaCO}_{2}$ of $79 \mathrm{~mm} \mathrm{Hg}$, and $\mathrm{PaO}_{2}$ of $119 \mathrm{~mm} \mathrm{Hg}$, and the ventilatory support included pressure support of $15 \mathrm{~cm} \mathrm{H}_{2} \mathrm{O}$, positive end-expiratory pressure (PEEP) of $10 \mathrm{cmH}_{2} \mathrm{O}$, and $\mathrm{FiO}_{2}$ of 0.50 . From then on, it was assumed that the tumor might be malignant, and there was no conclusive proof that we would be able to treat it radically. However, she was also considered to be on the verge of suffocation. So, we decided to immediately connect her to VV-ECMO. The ECMO was initially set as follows: blood flow $3.5 \mathrm{~L} / \mathrm{min}$ and sweep gas flow $5.0 \mathrm{~L} / \mathrm{min}\left(\mathrm{FiO}_{2}\right.$ 0.70 ). It was unnecessary to adjust the ECMO during its running period. After the initiation of ECMO, the general status was stabilized, while the tidal volume finally decreased to approximately $30 \mathrm{~mL}$. Pathological examination was implemented subsequently and she was diagnosed with large B-cell lymphoma. On the third day, prednisolone was administered and followed by R-CHOP regimen chemotherapy. The mediastinal tumor reduced promptly (Fig. 1c, d), and the trachea regained its patency dramatically (Fig. 2). On the 10th day, it became possible to ventilate both lungs. When the sweep gas was stopped on trial off, arterial blood gas test showed a pH of 7.48, a $\mathrm{PaCO}_{2}$ of $34 \mathrm{~mm} \mathrm{Hg}$, and a ratio of partial pressure arterial oxygen and fraction of inspired oxygen (P/F ratio) of 300. Then ECMO was weaned on the 12th day. Throughout the period, partial thromboplastin time (PTT) and activated clotting time (ACT) had been maintained 48-62 s and 150-180 s, respectively. Platelet counts decreased gradually, but it had never been below $8 \times 10^{4} / \mu \mathrm{L}$. Furthermore, no complication such as bleeding and infection occurred. After the discontinuation of ECMO, her respiratory status had been still stable, and arterial blood gas test showed a pH of 7.44, $\mathrm{PaCO}_{2}$ of $39 \mathrm{~mm} \mathrm{Hg}$, and a P/F ratio of 430 . She was extubated on the 16th day and discharged to the general ward on the 19th day 
without any sequelae. Chemotherapy was continued and she was discharged on the 40th day. Several courses of chemotherapy were followed. For the time being, she regularly attends a hematologist at our hospital and has been free from airway obstruction and a relapse so far.

\section{Discussion}

Central airway obstruction is a life-threatening pathology. The most common cause is direct extension from an adjacent tumor, such as bronchogenic carcinoma, esophageal carcinoma, and thyroid carcinoma. In addition, mediastinal tumor or nonmalignant etiologies are other possible causes [4]. It is reasonable that symptoms related to airway obstruction are in proportion to the extent of stenosis. However, it is also reported that patients do not show any symptom until the trachea is significantly narrowed to less than $8 \mathrm{~mm}$. Then, once the lumen is less than $5 \mathrm{~mm}$, symptoms present also at rest [4]. In this case, the narrowest diameter of the trachea was about $4 \mathrm{~mm}$, which was just above the carina. That was narrow enough to cause dyspnea.

For patients with central airway obstruction, no matter what the cause is, securing the airway is essential. Especially in unstable patients, it might be challenging and should be accomplished by the most experienced physician. The strategy differs depending on the location and the extent of stenosis. Proximal airway obstruction simply requires tracheal intubation or tracheostomy. However, in the case of more distal obstruction, tracheal intubation might not be sufficient for ventilation. In such situations, ECMO is assumed to be used as a bridge until the procedures leading to definitive airway security are implemented $[5,6]$.

ECMO is a remedy against severe heart or lung failure refractory to conventional management $[7,8]$. Respiratory ECMO was initiated for neonates and pediatrics in the 1970's, and recently it has become widespread for adult patients through the publication of the CESAR trial [8] or the successful experiences of managing the influenza H1N1 pandemic. It is often the case that respiratory ECMO is used for patients with oxygenation or ventilation failure, such as severe ARDS [7], and on the other hand, airway obstruction is relatively uncommon as an indication of ECMO.

Previous studies reported that ECMO should be applied carefully for hematological malignancy patients. Gow et al. [1] analyzed the data from the ELSO Registry and concluded that survival is decreased among adult patients with cancer compared with those without cancer. Moreover, the development of infections was considered an indicator for poor prognosis. Wohlfarth et al. [2] reported the case series of hematological malignancy patients managed with ECMO and concluded that ECMO was feasible in this population as well. However, it was also stated that these patients were at increased risk for severe bleeding events. Kang et al. [9] reported that case series of hematological malignancies supported by ECMO and bleeding events were not significantly more frequent in those patients than in immunocompetent patients. Thus, hematological malignancy patients have several risks related to ECMO management such as bleeding and infection. In order to manage successfully, it seems essential to weigh the risks appropriately and to avoid these complications. The reason why we could manage this case without any complication might be that chemotherapy brought an immediate result and ECMO could be disconnected before WBC nadir, which made us avoid the risks of bleeding or infection (Fig. 2). Regarding the indication of ECMO for hematological malignancy patients, physicians have to consider it carefully, but do not have to regard it as a contraindication. According to the ELSO guidelines, most contraindications are relative, balancing 
the risks of the procedure against the potential benefits [10]. As a matter of course, in the case of imminent suffocation like our case, it is inevitable to initiate ECMO to save life.

In conclusion, we experienced a case of airway obstruction related to malignant lymphoma successfully managed with VV-ECMO. ECMO can be a treatment choice also for hematological malignancy patients, but physicians should pay careful attention to hemorrhagic complications and the development of infection.

\section{Statement of Ethics}

The authors have no ethical conflicts to disclose. Informed consent was obtained from the patient for publication of this case report.

\section{Disclosure Statement}

The authors have no conflicts of interest to declare.

\section{Funding Sources}

The authors did not receive any financial support.

\section{References}

1 Gow KW, Lao OB, Leong T, Fortenberry JD. Extracorporeal life support for adults with malignancy and respiratory or cardiac failure: The Extracorporeal Life Support experience. Am J Surg. 2010 May;199(5):669-75.

2 Wohlfarth P, Ullrich R, Staudinger T, Bojic A, Robak O, Hermann A, et al.; Arbeitsgruppe für hämatoonkologische Intensivmedizin der Österreichischen Gesellschaft für Internistische und Allgemeine Intensivmedizin und Notfallmedizin (ÖGIAIN). Extracorporeal membrane oxygenation in adult patients with hematologic malignancies and severe acute respiratory failure. Crit Care. 2014 Jan;18(1):R20.

3 Ernst A, Feller-Kopman D, Becker HD, Mehta AC. Central airway obstruction. Am J Respir Crit Care Med. 2004 Jun;169(12):1278-97.

4 Chang YC, Lee JM, Ko WJ, Lee YC. Airway obstruction following bronchoscopic photodynamic therapy in early centrally located lung cancer requiring extracorporeal membrane oxygenation. J Formos Med Assoc. 2013 Jan;112(1):54-6.

5 Makdisi G, Wang IW. Extra Corporeal Membrane Oxygenation (ECMO) review of a lifesaving technology. J Thorac Dis. 2015 Jul;7(7):E166-76.

6 Peek GJ, Mugford M, Tiruvoipati R, Wilson A, Allen E, Thalanany MM, et al.; CESAR trial collaboration. Efficacy and economic assessment of conventional ventilatory support versus extracorporeal membrane oxygenation for severe adult respiratory failure (CESAR): a multicentre randomised controlled trial. Lancet. 2009 Oct;374(9698):1351-63.

7 Hong Y, Jo KW, Lyu J, Huh JW, Hong SB, Jung SH, et al. Use of venovenous extracorporeal membrane oxygenation in central airway obstruction to facilitate interventions leading to definitive airway security. J Crit Care. 2013 Oct;28(5):669-74.

8 Brodie D, Bacchetta M. Extracorporeal membrane oxygenation for ARDS in adults. N Engl J Med. 2011 Nov;365(20):1905-14.

9 Kang HS, Rhee CK, Lee HY, Kim YK, Kwon SS, Kim SC, et al. Clinical outcomes of extracorporeal membrane oxygenation support in patients with hematologic malignancies. Korean J Intern Med (Korean Assoc Intern Med). 2015 Jul;30(4):478-88.

10 Extracorporeal Life Support Organization (ELSO) General Guidelines for all ECLS Cases. August, 2017. 


\section{Case Reports in Acute Medicine}
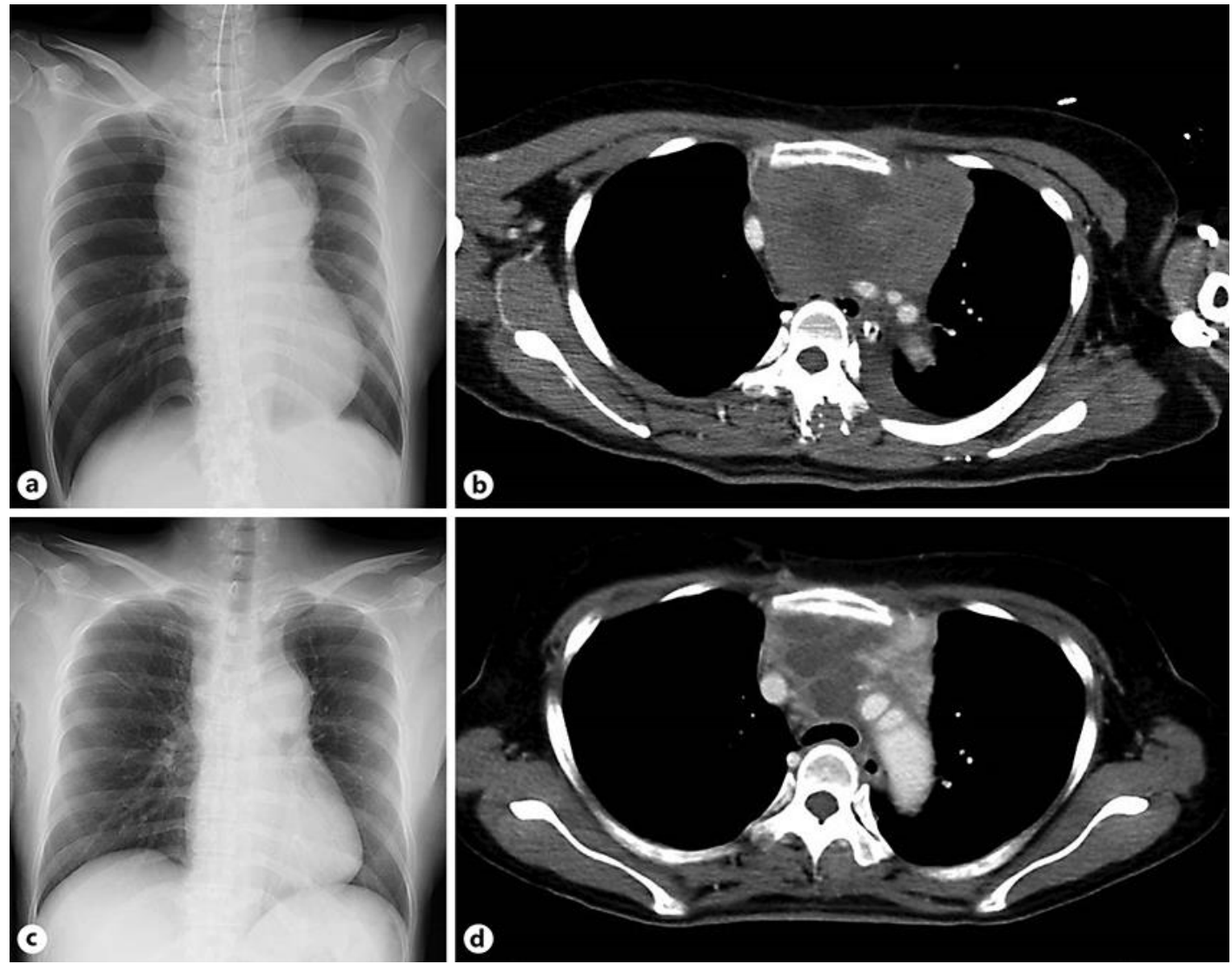

Fig. 1. Chest X-ray and CT scan on days 2 (a, b) and 19 (c, d). a Chest X-ray indicating a mediastinal mass. b CT scan showing that the trachea is compressed by an anterior mediastinal tumor. c Chest X-ray indicating a downsized mediastinal mass. $\mathbf{d}$ CT scan showing that tumor is reduced and the trachea regains its patency. 


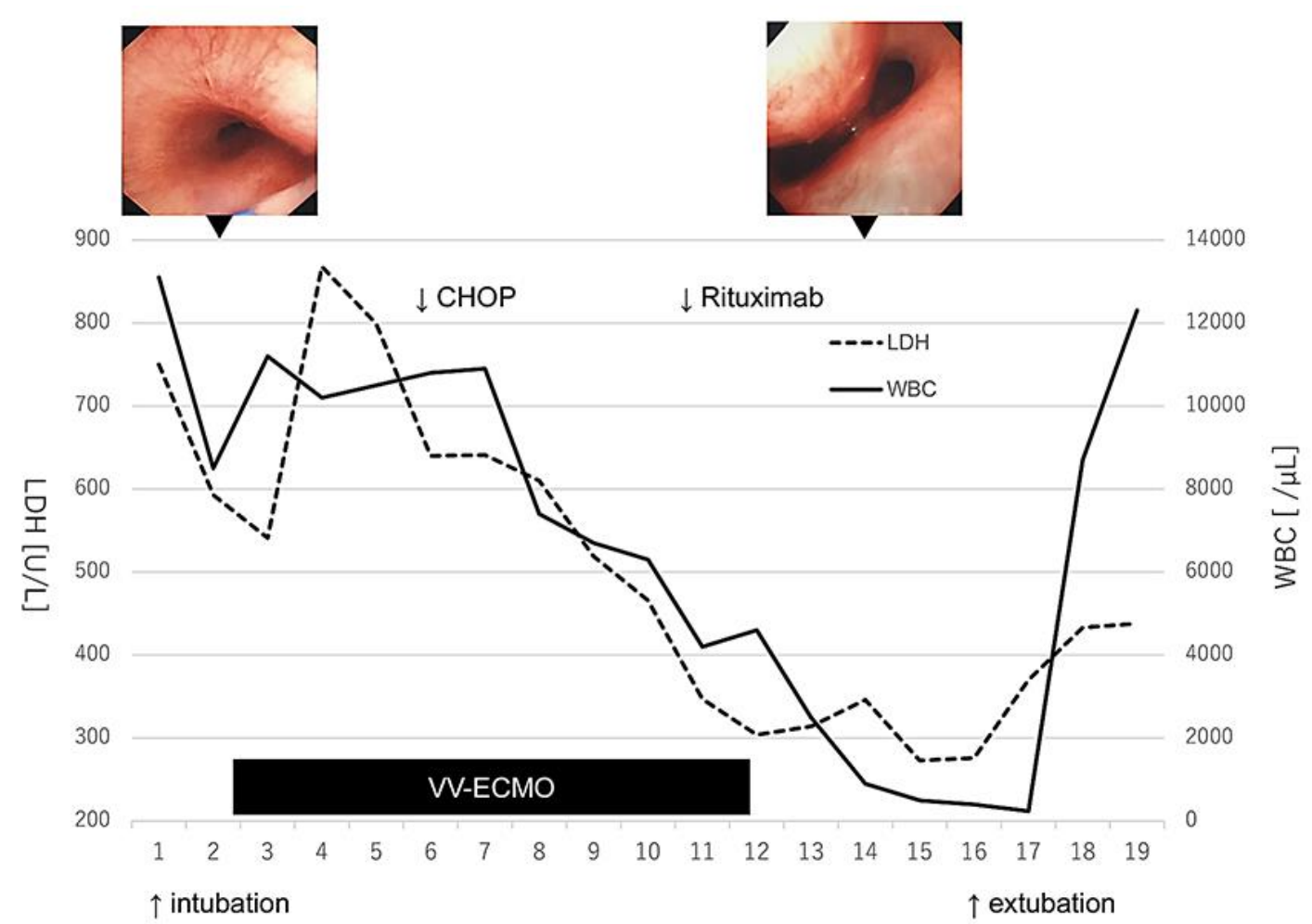

Fig. 2. The clinical course during ICU stay and bronchoscopic findings on days 2 and 14 (upper 2 photographs). Chemotherapy brought an immediate result and ECMO was disconnected before WBC nadir. The value of LDH decreased dramatically, which was correlated with the reduction of the tumor. 